\title{
A Survey on Sentiment Analysis
}

\author{
Megha Joshi \\ Charotar University of \\ Science \&Technology \\ Changa, Gujarat \\ India
}

\author{
Purvi Prajapati \\ Charotar University of \\ Science \&Technology \\ Changa, Gujarat \\ India
}

\author{
Ayesha Shaikh \\ Charotar University of \\ Science \&Technology \\ Changa, Gujarat \\ India
}

\author{
Vishwa Vala \\ Charotar University of \\ Science \&Technology \\ Changa, Gujarat \\ India
}

\begin{abstract}
Sentiment analysis is process of extracting information from user's opinions. Every person shares his or her information on social network sites, blogs, product review websites and webforums. Thus, we get familiar with the thinking of the other people. People's thinking that provides an information that helps in decision making process. This Paper describe different applications of sentiment analysis, techniques and challenges of sentiment analysis.
\end{abstract}

\section{Keywords}

Sentiment analysis, classification, Machine Learning

\section{INTRODUCTION}

\subsection{Introduction}

Estimation investigation is a data gathering assignment to accomplish client's sentiments. Utilizing opinion investigation Researchers can examining huge quantities of archives, these sentiments can be communicated into various way positive, negative and neutral routes as remarks, inquiries and solicitations. ${ }^{[1,2,4]}$

Generally, sentiment analysis is classification of the give text polarity in these three levels sentence Level, Document level or Aspect level. Fundamental point of sentiment analysis is to decide the mentality of creator or speaker with respect to some subject or overall polarity of an opinion. Because of the exponential upgrade in the Internet usage and substitution of popular conclusions, opinion examination turns into a vital procedure in today's life. For ordered and unstructured data The Web is a huge depository. ${ }^{[1]}$

Assumption investigation should be possible at three levels that are document level, sentence level and Aspect level. ${ }^{[1]}$ Sentiment analysis is additionally called opinion extraction, opinion mining, sentiment mining, affect analysis, review mining, emotion analysis etc. ${ }^{[1]}$ these are the many names of it and slightly different tasks as per their name. ${ }^{[1]}$

Sentiment analysis is field of study that investigation of individuals assessment, estimations, disposition and emotions towards entities for example items, services organizations individual events issues, subjects and their attributes. ${ }^{[3]}$

\subsection{Challenges}

Twitter have reported everything from daily life story to real word event. Millions of tweet updated so people have no time to visualize all those tweet.

A major problem is there is no any restriction to post a tweet, update information or status so many people provide false, incorrect information about some events. Large number of spellings and grammar error, and the use of not a proper sentence structure and mixed language so people can't distinguish important data from unused data. Not all tweets are relevant to the user query or interest profile.
One way communication. Twitter often acts as a one-way communication platform. Twitter used by celebrities, TV shows, companies and websites to simply get the word out. It is not used for relationship building.

\section{DIFFERENT LEVELS OF SENTIMENT ANALYSIS}

Different three levels in sentiment analysis which is document level, sentence level and aspect level. In document level i.e. identified that is the review is positive or negative. In sentence level i.e., identified every sentence is positive or negative and in aspect level entities and their features/aspects Sentiments is positive and negative. ${ }^{[2]}$

\subsection{Document level}

In Document level analysis task is characterize whether an entire opinion of document level communicates a positive or negative supposition For instance, given thing audit, the framework figures out if the survey communicates a general positive or negative decision about anything. This undertaking is regularly known as document level sentiment classification.

\subsection{Sentence level}

In Sentence level the fundamental undertaking is goes to the Sentence and makes sense of if every sentence communicated a positive, negative, or neutral sentiment. Neutral means no opinion about any sentence. This level of investigation is immovably related to the subjectivity arrangement. which is recognizes sentences (called target sentences) ${ }^{[2]}$ that is express genuine information from the sentences (called subjective sentences) that express subjective perspectives and opinions.in any case, we ought to observe that subjectivity is not comparable to supposition the same number of target sentences can suggest feelings for e.g., "We purchased new car a month ago and the windshield wiper has tumbled off". ${ }^{2}$, 15]

\subsection{Aspect level}

In Aspect Level both the document level and the sentence level analyses do not discover what exactly people liked and didn't like. Aspect level performs better-grained investigation. Aspect level is directly looks at the opinion itself. In the Aspect level is depend on the possibility that an opinion consists of a sentiment positive, negative or neutral or an objective of sentiment. ${ }^{[2,15]}$

For e.g. Sentence is "The Sony telephone's call quality is amazing, yet its battery life is short" assesses two focuses first is call quality second is battery life, of Sony (component). The conclusion on Sony's call quality is certain in sentence however the opinion on its battery life is negative. Sony telephone's call quality and battery life of Phone are the feeling targets. In this level of investigation, an organized of assessments about elements and their viewpoints can be created, which turns unstructured content to organized 
information and can be utilized for a wide range of quantitative and subjective examinations. Document and sentence levels orders are as of now very difficult. ${ }^{[2,15]}$

\section{SENTIMENT ANALYSIS TECHNIQUES}

Sentiment Classification techniques are separated into two different techniques which is ML and Lexicon based Approaches. ${ }^{[1,2,4,7]}$

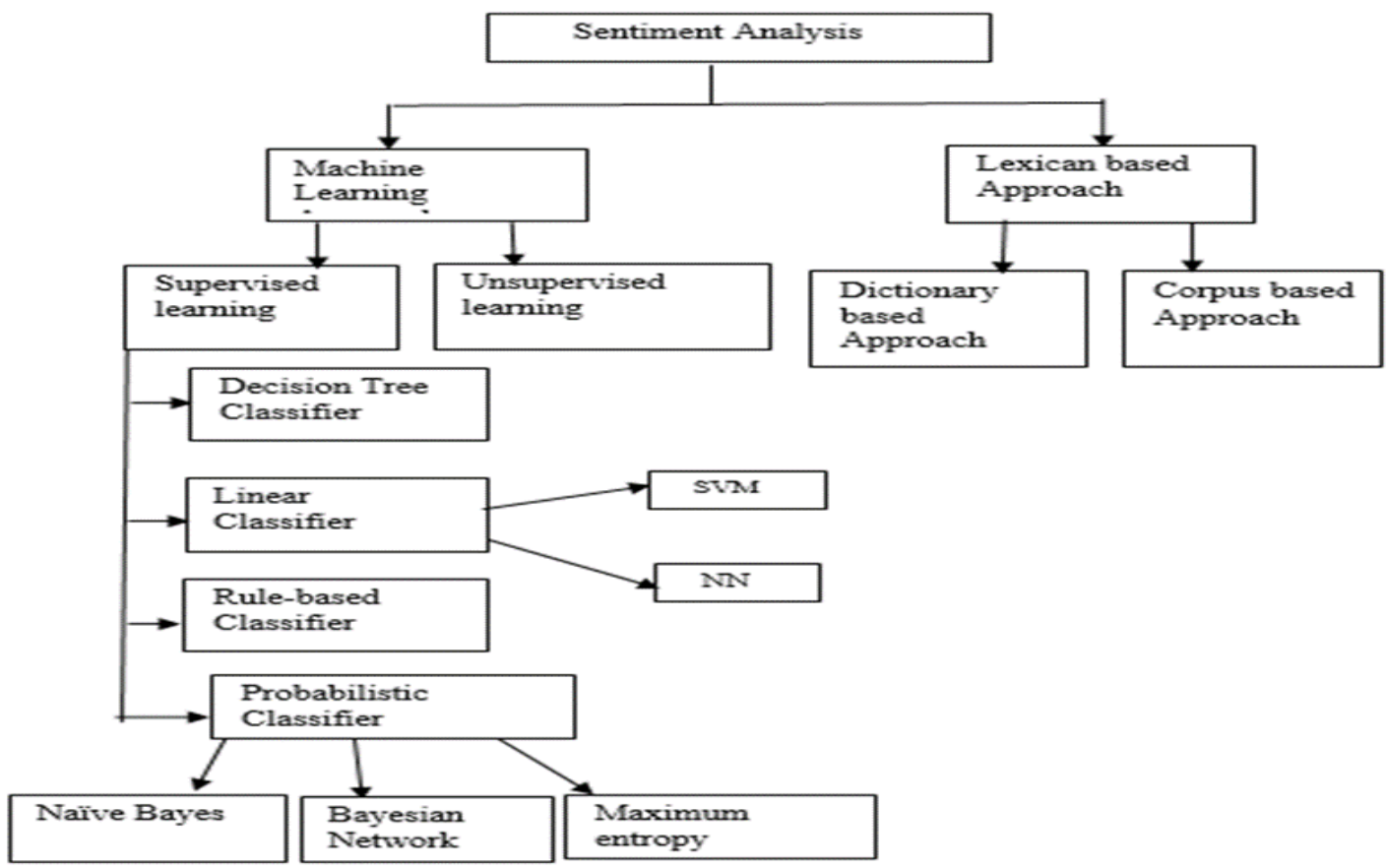

Fig 1 Sentiment Classification techniques

\subsection{Machine Learning approach for SA}

Machine learning approach is relies on upon ML algorithms to unravel the Sentiment Analysis as a standard substance classification issue that makes use of syntactic as well as linguistic features. ${ }^{[1,3,7,11]}$

\subsubsection{Supervised learning}

In the supervised learning technique it is relay upon the presence of labeled Dataset and along these labels are given to the model amidd the procedure there are different supervised learning model. ${ }^{[1,3,7,8]}$

\subsection{Decision tree classifiers}

This classifier gives a various leveled deterioration of the preparation information space in which a condition on the credit esteem is used to saprate the data. [1] The data division space is done recursively until the leaf hubs contain certain base quantities of records which are utilized with the end goal of characterization. The choice tree executions in content characterization have a tendency to be minimal minor departure from standard bundles for instance as ID3 and C4.5.

\subsection{Linear classification}

There are different sort of Linear classifiers among them is Support vector machine which is a kind of classifier that attempt to choose awesome direct separators between different classes and another technique is neural system. ${ }^{[1,7,9]}$

SVM is supervised learning model. Support Vector Machines work on idea of decision planes that specify decision boundaries. Set of objects belonging to various class memberships are participations by decision planes. Shown in
Figure example to illustrate the concept of linear SVMs In the objects either belong to GREEN class (or RED class) in this example.

Isolated line determine the choice. On the correct hand side of the boundary, all objects are GREEN and to the side hand side of boundary, all articles are RED. A new object white circle will be classified as GREEN if it falls to the correct hand side of the boundary or classified as RED if it falls to the one side of the boundary.

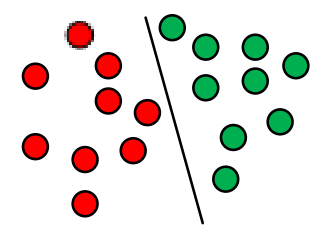

Fig 2(A) Example of Linear SVM

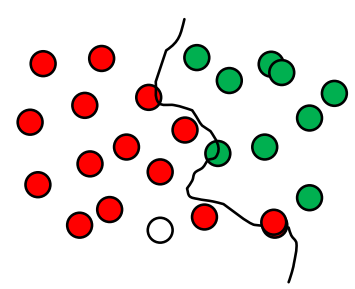

Fig 2(B) Example of Hyperplane SVM

A classifier partitions a set of objects into their respective domains with a line is called linear classifier and partitioning with a curve is known as hyperplane classifier. An example of hyperplane classifier is shown in figure. 
Fig. demonstrate the basic idea behind Support Vector Machines. The figure shows that the mapped objects are linearly separable. Thus, find an optimal line rather than constructing the complex curve that can divide the GREEN and the RED objects.

\section{INPUT SPACE $\quad$ FEATURE SPACE}

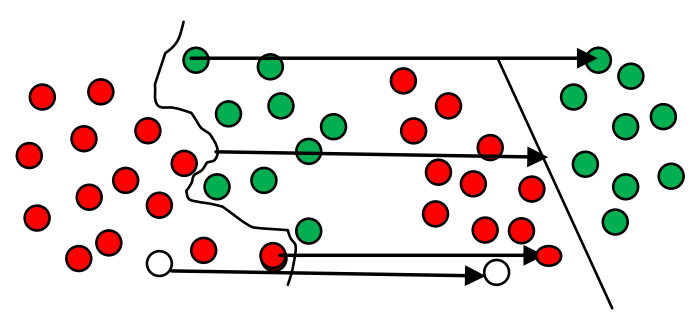

Fig 2(C) mapping of objects in SVMs

SVM has portrayed information and yield organize the Input is a vector space and yield is 0 or 1 (positive/negative). Content archive in unique shape are not reasonable for learning. Archives are changed into configuration which matches into contribution of machine learning calculation input. For this pre-preparing on content archives is done. At that point carryout transformation.[10] Every word will relate to one measurement and indistinguishable words to same measurement. SVM has been demonstrated one of the viable learning calculation for content classification. ${ }^{[10]}$

\subsection{Rule-based Technique}

In rule based technique, if a rule has "if-then" relation then it consists of an antecedent and its associated consequent. [10,11, $12,16]$

\section{Antecedent $\rightarrow$ consequent}

An antecedent describes a condition and can be either a token or a series of tokens that are concatenated by the " $\wedge$ " operator. A token can be either '?' denoting a proper noun, a word or '\#' denoting the result of the condition described by the antecedent

$\{$ token $1 \wedge$ token $2 \wedge \ldots \wedge$ token $\} \Rightarrow\{+\mid-\}$

The two simple rules $\mathrm{A}$ and $\mathrm{B}$ depend on two sentiment bearing words, each of which denotes an antecedent.

$\{$ Excellent $\} \Rightarrow\{+$ ve $\} \ldots$ (A)

$\{$ Absurd $\} \Rightarrow\{-\mathrm{ve}\} \ldots$ (B)

Assume two sentences

I) Mobile-A is more expensive than Mobile-B

II) Mobile-A is more expensive than Mobile-C

The target word of sentences (I) and (II) is Mobile-A. The rule deduced from these sentences is as given in (III).

$\{\# \wedge$ more $\wedge$ expensive $\wedge$ than $\wedge$ ? $\} \Rightarrow\{-\}$..

The analysis of this rule is as the target word, i.e., Mobile-A is less favorable than the other two mobiles because of its price that is expressed by the rule (III). Here, the center of attention is on the price attribute of the Mobile-A. ${ }^{[1,10,16]}$

\subsection{Probabilistic classifiers}

Probabilistic classifiers use blend of models for the grouping. [1] Mixture demonstrate expect that each class is a portion of the blend. Each blend fragment is a generative model that gives the likelihood of examining a particular term for that segment. [4] These sort of classifiers are additionally called generative classifiers. There are three probabilistic classifiers which are Naïve Byes, Maximum Entropy and Bayesian Network. ${ }^{[1,3,4]}$

\subsubsection{Naive Bayes Classifier (NB)}

NB classifier is a probabilistic classifier it is rely on upon Bayes hypothesis with solid and innocent freedom suppositions. ${ }^{[1]}$ It is champion among the most principal content order procedures with different applications archive classification, email spam discovery, individual email sorting, slant recognition and dialect identification. It's performs better in numerous perplexing genuine issues. ${ }^{[1]}$

These are the Naive Bayes Variations which is Multinomial Naive Bayes another is Binarized Multinomial Naive Bayes and the Bernoulli Naive Bayes. Each framework pass on very surprising result since they used absolutely one of a kind models. When the different events of the words matter a great deal in the characterization issue Multinomial Naive Bayes is utilized. Binarized Multinomial Naive Bayes used when the frequencies of the words don't accept a key part in our arrangement.

\subsubsection{Bayesian Network (BN)}

The Naïve Bayes classifier is the independence of the features. Assumption of Naïve Bayes is to expect that every one of components are completely dependent. ${ }^{[1]}$ This prompts to the Bayesian Network shows which is a coordinated acyclic graph and whose nodes correspond random variables, and edges represent conditional dependencies. ${ }^{[1]}$

Bayesian Network viewed as an entire factors and their association. In this way, an entire joint probability distribution over each one of the element is resolved for a model. The computation complexity of the $\mathrm{BN}$ is exceptionally costly in text mining; so, BN is very little of time used. Bayesian Network was utilized to consider a true issue.

\subsubsection{Maximum Entropy Classifier (ME)}

It is a probabilistic classifier which has a place with the class of exponential models. Most extreme Entropy does not expect that the components are individually free of each other. The Maximum Entropy is relies on upon Principle of Maximum Entropy and from each one of the models that fit preparing information, pick the one which has the greatest entropy. The Max Entropy classifier can be used to illuminate a vast assortment of content characterization issue, for example, dialect identification, point arrangement, assumption investigation and more. ${ }^{[1]}$

\subsubsection{Unsupervised Techniques}

In unsupervised technique sentiment classification is finished by comparison. In this technique, the components of a given content are compared against word lexicons whose sentiment values are chosen prior to their use ${ }^{[1,3]}$. Hierarchical clustering and partial clustering are mostly utilized algorithms of unsupervised technique. Both algorithms are discussed as follows. ${ }^{[7,8]}$

\subsubsection{Hierarchical Clustering}

Hierarchical clustering algorithms partition the objects into tree like structure where each node represents a cluster. There are zero or more child nodes in each node of the tree. 


\subsubsection{Partial Clustering}

In partial clustering algorithm, objects are partitioned. Objects can change the clusters on the basis of dissimilarity. K-mean Clustering algorithm is mostly used algorithm of partial clustering algorithm.

\subsection{Lexicon-based approach}

This approach is depends on sentiment Lexicons.

Lexicon considers as an important indicator for sentiment, which called opinion word. Lexicon separated into dictionary approach and corpus based approach. ${ }^{[1,3]}$

\subsubsection{Dictionary-based approach}

An arrangement of sentiment word is gathered manually with known instructions. The conclusion set is created by means of looking in the notable corpora WordNet for their proportionate word and antonyms ${ }^{[1,14,15]}$. The next iteration starts when the words are added in to the seed list. The iterative strategies stops exactly when no new words are found. After the system completed, manual appraisal can be done to evacuate or amend errors. ${ }^{[1]}$ Dictionary approach has an inconvenience that is the inability to discover feeling words with space and setting specific introductions. ${ }^{[1,14,15]}$

\subsubsection{Corpus-based approach}

Corpus-based approach is deals with the issue of finding feeling words with setting specific presentation. Corpusconstruct techniques depend in light of upon syntactic illustrations or examples that happen together alongside a seed rundown of assessment words to discover other conclusion words in a broad corpus. The objectives are for connectives like AND, OR, BUT, EITHER E-XOR ${ }^{[1]}$. The conjunction in AND case that are conjoined descriptive words for the most part have the comparative introduction. This contemplation is called notion consistency, which is not for the most part solid for all predictable practically. ${ }^{[1]}$ There are also adversative expressions on account of BUT, however which are shown as opinion changes. So as to figure out whether two conjoined descriptors are of the same or distinctive introductions. Corpus-based approach is use factual or semantic techniques to discover assumption extremity.

\section{APPLICATIONS OF SENTIMENT ANALYSIS}

There different application of Sentiment Analysis. Sentiment analysis used in the movie review, product review, politics, public sentiment and social sites useful for people's opinion. $[1,2,3,4]$

Shown in the table there are various application of sentiment analysis in movie review by this user can get information about movie is good or bad or average by their star scale rating if movie is five stare we can predict that movie will be good if three star it will average review of movie. ${ }^{[2]}$

From the product review user can identify that product is good, excellent, average, and poor with the public opinion by their rating. When user have to settle on a choice user need to know others opinion. In the organization and associations dependably need to discover shopper or general assessments about their items and administration.
Table 1. Application OF SA

\begin{tabular}{|l|l|}
\hline $\begin{array}{l}\text { Different } \\
\text { application }\end{array}$ & Different rating \\
\hline Movie review & \\
\hline Product review & \\
\hline Politics & \\
\hline Public sentiment & \\
\hline
\end{tabular}

Mostly in the business or any affiliation required open or purchaser felling, it directed review, opinion polls, and focus on groups. The explosive growth of social media for example Twitter, Facebook, remarks, furthermore, posting in informal community destinations on the Web. Overviews, online journals, web journals ${ }^{[4]}$ Sentiment analysis applications have spread to each conceivable space, items, administrations, human services, and budgetary administrations to gettogethers and political decisions. These applications gave inspirations to inquire about in conclusion examination.

\section{CHALLENGES OF SENTIMENT ANALYSIS}

There are different challenges in sentiment analysis which is describe below. ${ }^{[14]}$

\subsection{Implicit Sentiment and Sarcasm}

There is a chance that a sentence may contain implicit sentiment even though it is not having any word that earns sentiments. For an example, two statements are taken; "One should question that the stability of mind of the writer who wrote this book". In this above sentences don't have negative sentiment bearing words and no negative words are seen, although both are negative sentences. Thus identifying sentiment is important in Sentiment Analysis than syntax detection. ${ }^{[15]}$

\subsection{Domain Dependency}

In this type of challenge words polarity changes from one domain to another domain in the domain dependency. For an example, two statements; "The story was unpredictable." and "The steering of car is unpredictable." In first statement, in

Sentiment express that is positive whereas the second statement express sentiment is negative. ${ }^{[15]}$

\subsection{Language Problem}

In Opinion Mining English language is mostly used because of its resources availability means lexicons, dictionaries and corpora but User get attracted by using Opinion mining with language other than English like Hindi, French, Chinese, and 
German Arabic etc. i.e. lexicons dictionaries and corpora for these languages. ${ }^{[14]}$

\subsection{Fake Opinion}

Fake opinion is also called fake review and refers to bogus or fake reviews. The fake opinion is misguide the users or readers by providing them untruthful positive or negative opinion related with any object. This is social challenge which is faces by OP. ${ }^{[14]}$

\subsection{Negation}

In Sentiment Analysis Negation is Challenging task. Invalidation is communicated from multiple points of view without the reasonable seen utilization of any negative word in the sentence. The presence of a negation is usually change the opinion polarity. For example she is not happy. ${ }^{[15]}$

\section{CONCLUSION}

Sentiment analysis is helps in identifying people's emotional and attitudes states. People's feeling that can be expressed in positive or negative ways. This paper talks about in subtle elements the different ways to deal with sentiment Analysis, mostly ML and Lexicon-based approaches. This survey paper is gives a point by point perspective of the distinctive applications and challenges of Sentiment Analysis. Sentiment analysis can be extremely compelling in foreseeing decision comes about, securities exchange or motion picture survey like Imdb audits of facebook and twitter can be likewise used to give helpful information which can be utilized to anticipate future.

\section{REFERENCES}

[1] Walaa Meddhat , Ahmed Hassan ,Hoda Korashy "Sentiment analysis algorithms and applications: A survey, Ain Sham University, Faculty of Engineering, Computer \& Systems Department, Egypt 19 April 2014.

[2] Xing Fang and Justin Zhan "sentiment analysis using product Review data" Department of computer science, North Carolina a\&T State University Greensboro, NC, USA, 2015 Springer journal.

[3] Ebru Aydogan and M. Ali Akcayol "A Comprehensive Survey for Sentiment Analysis Tasks Using Machine Learning Techniques" Department of Computer Engineering Gazi University Ankara, Turkey 2016 IEEE

[4] Bogdan Batrinc, Philip C. Treleaven "Social media analytics: a survey of techniques, tools and platforms Department of computer science, University College London, Grower Street, London WCIE 6BT, UK Published on 26 July 2014.

[5] Soo-Min Kim, Eduard Hovy, "Determining the Sentiment Opinions", Information Sciences Institute University of Southern California Marinadel Rey, CA 90292-6695, 2004.
[6] Hong $\mathrm{Yu}$, Vasileios Hatzivassiloglou, "Towards Answering Opinion Questions: Separating Facts from Opinions and Identifying the Polarity of Opinion Sentences" Department of Computer Science Columbia University, Newyork, NY 10027, USA.

[7] Federico Neri Carlo Aliprandi Federico Capeci Montserrat Cuadros Tomas "Sentiment Analysis on Social Media" IEEE/ACM 2012

[8] Tan Li Im, Phang Wai San, Chain Kim On, Center of Excellence in Semantic Agents, University Malaysia, Patrica Anthony, Department of Information and Enabling Technologies, Faculty of Environment, Society and Design, Linclon University, Christchruch, New Zealand "Rule-based Sentiment Analysis for Financial News, 2015 IEEE .

[9] Ankush Sharma, Aakanksha, Assistant Professor, Department of C.S.E, Chandigarh University Gharuan, India, International journal of Advanced Research in Computer and Communication Engineering, " A Comparative Study Of Sentiments Analysis Using Rule Based and Support Vector Machine " volume 3, March 2014 .

[10] Chin-Shrng Yang, Hsiao-Ping Shih, Department of Information Management, Yuan Ze University, ChangLi, Taiwan," A Rule-Based Approach For Effective Sentiment Analysis" PACIS 2012.

[11] Prern Chikersal, Soujanya Poria, and Erik Cambria, School of Computer Engineering Nanyang Technological University Singapore-639798,“ SeNTU: Sentiment Analysis of Tweets By Combining a Rulebased Classifier with Supervised Learning" June 52015.

[12] Neethu M S Rajasree R Department of Computer Science and Engineering "Sentiment Analysis in Twitter using Machine Learning Techniques" IEEE 2013

[13] Geetika Gautam Divakar yadav Department of Computer Science \& Engg. Sentiment Analysis of Twitter Data Using Machine Learning Approaches and Semantic Analysis IEEE 2014

[14] Chandni, Nav Chandra, Sarishty Gupta, Renuka Pahade MSc[Eng] (Software Engineering), The University of Sheffield, United Kingdom "Sentiment Analysis and its Challenges" International Journal of Engineering Research \& Technology (IJERT) 2015

[15] Vishal A. Kharde S.S. Sonawane Department of Computer Engg, Pune Institute of Computer Technology, Pune University of Pune (India) "Sentiment Analysis of Twitter Data: A Survey of Techniques" International Journal of Computer Applications April 2016

[16] Sujata Rani and Parteek Kumar "Rule Based Sentiment Analysis System” Elsevier Publications 2014. 